$\mathrm{Zu}$ den brennendsten Problemen der Nord-Süd-Beziehungen gehört seit einigen Jahren die Verschuldung des größten Teils der Dritten gegenüber der Ersten und Zweiten Welt. Verwaltung und Lösung dieser Problematik lagen bisher wesentlich in den Händen von Wirtschafts- und Finanzexperten, wobei die großen Gläubigernationen, koordiniert durch den Internationalen Währungsfonds, es verstanden haben, Richtung, Tempo und Inhalt der Debatten zu dominieren. Internationales Recht - im weiteren Sinne des Begriffs - und Rechtswissenschaft, die aus den NordSüd-Beziehungen im allgemeinen wesentliche neuere Entwicklungsimpulse empfangen haben, spielten in diesem setting eine eher bescheidene Rolle. ${ }^{\mathrm{I}}$ Der wesentliche juristische Beitrag lag neben einer systematisierenden Arbeit in Überlegungen zu legitimationsstiftenden Verfahren. Die materieilen Aspekte der Gläubiger-Schuldner-Beziehungen blieben bisher weitgehend aus der Betrachtung ausgespart. Dabei sind sowohl die Gläubiger als auch die Schuldner als auch die Beziehungen ganz besondere und wert, eingehend und im Detail juristisch analysiert zu werden.

Der folgende Diskussionsbeitrag verfolgt die Absicht, in diese Debatten um Versçhuldung und Technologie-Transfer in die Dritte Welt eine juristische Dimension einzuführen, die wesentlich für nationale Rechtssysteme entwickelt worden ist und dort dazu beiträgt, sowohl die Probleme einzelner Schuldenverhältnisse als auch die der (drohenden) Zahlungsunfähigkeit eines Schuldners zu lösen. Die diskutierten Doktrinen und Lösungen sind weder sonderlich orginell noch gar umwälzend, sondern haben sich den Notwendigkeiten einer Waren-Geld-Gesellschaft entsprechend entwickelt. Überraschend und originell mag lediglich erscheinen, wie weit die im Zivil- und Handelsrecht absolut geläufigen Doktrinen die internationale Schuldendebatte in eine neue Richtung drängen können.

Bevor Lösungen im einzelnen vorgetragen werden, müssen einige Präzisierungen und Hypothesen vorgestellt werden, die den Diskussionsrahmen bestimmen. Die Vorschläge beziehen sich auf eine Situation, die gemeinhin als technologie-transfer-trächtig umschrieben wird. Das heißt, es wird von Ländern der Dritten Welt ausgegangen, die wesentlich Objekt von Industrialisierungsprozessen sind und eine Gütereinfuhrstruktur yorweisen, die sich aus dem Stand der nationalen Technologie-Entwicklung ergibt. Um es konkreter zu sagen, das können schlüsselfertige Fertigungsanlagen sein sowie komplizierte Waffen, Medikamente, optische Geräte etc.

Es wird die weitere Vorgabe gemacht, daß ein Staat sich etwa ein schlüsselfertiges Unternehmen planen und errichten läßt, weil im Land selbst keine (technische) Kapazität existiert, die aus eigener Anstrengung dieselbe Leistung vollbringen könnte. Dies trifft sicherlich auf die meisten der Länder zu, die in der großen

I Vgi. erwa Carreau, Le rééchelonnement de la dette extérieure des Etats, i 12 Journal du Droit International 1985 , S. 5 ff., Horn, Rechtsfragen internationaler Umschuldungen, WM 1984, S. 713 ff., Frankenberg/ Knieper, Rechtsprobleme der Überschuldung von Ländern der Dritten Welt, RIW 1983, S. 569ff. 
Dekolonisierungswelle der Nach-Weltkriegszeit in die politische Unabhängigkeit entlassen worden sind, ohne daß eine einigermaßen ausgebildete Schicht von Technikern im weitesten Sinne vorhanden gewesen wäre. Sie müssen sich aus diesem Grunde ganz Technikern der Liefer-Länder anvertrauen, und zwar was die ingenieurmäßige, technische, ökonomische, finanzielle, juristische Planung angeht wie auch die Durchführung, das Management und die Kommerzialisierung.

Des weiteren wird vor der - im übrigen wenn auch mit verschiedenen Nuancen weitgehend akzeptierten - Hypothese ausgegangen, daß dann, wenn der Staat als ökonomischer Agent aufritt und etwa mit privaten kommerziellen und industriellen Unternehmen verhandelt, Grundsätze des Privat- und Handelsrechts anzuwenden sind. Dabei wird allerdings nicht die platte und imaginäre These von privater Autonomie und Willensfreiheit des Staates zum Maßstab der Dinge gemacht, sondern die moderne juristische Doktrin, die zwar die Willensfreiheit nicht vollständig beseitigt, aber doch aus ökonomischen, sozialen und anderen Rücksichten vielfältig einschränkt.

Der Beitrag akzeptiert die heute beinahe allgemein verbreitete Auffassung, daß die beiden abgelaufenen sog. "Entwicklungsdekaden« äußerst magere Ergebnisse erbracht haben. Diese Aussage kann bisher nicht hinreichend quantifiziert werden (tatsächlich ist es hohe Zeit, eine Land-für-Land-Analyse der Schuldenstruktur durchzuführen, aus der sich ergeben müßte, mit welchen Prognosen, Annahmen und Preisen Projekte geplant worden sind, wie Feasibility-Studien aussehen, und wie diese im "Norden « durchgeführten Studien von der Realisierung abweichen). Gleichwohl wird die These gewagt, daß eine große Anzahl der über staatliche Kreditaufnahme oder Garantien finanzierten Projekte wenigstens in den am wenigsten entwickelten Ländern (LLDCs) so schlecht laufen, daß sie nicht einmal die Fonds für den Schuldendienst generieren, geschweige denn rentabel wären, und daß ein großer Anteil der in diese Länder exportierten Waren nicht für den Aufbau von Exportindustrien verwendet werden. In der Konsequenz bleibt es dabei, daß die LLDCs kaum importsubstituierende Fertigungen aufgebaut haben und der ImportSchuldendienst nicht aus den Exporten von Industrieerzeugnissen, sondern wie zur Kolonialzeit aus Rohstofferlösen bestreiten müssen, eine - wie die Gegenwart zeigt - hoch konjunkturanfällige und manipulationsträchtige Situation.

Diese Aussagen werden wohl wenig bestritten. Sie bilden aber die Grundlage für erbitterte Polemiken, die sich um die Verantwortungs- und Schuldzuweisungen für diese Situation drehen. Da die Feindbilder häufig hochmoralisierend überzeichnet werden: hier korrupte Eliten, dort Neo-Imperialisten, besteht eine verständliche Tendenz, die gesamte Debatte um Verantwortlichkeit und Schuld fallen zu lassen und sich sog. objektiven ökonomischen Analysen zu verschreiben. Diese Ansicht schüttet das Kind mit dem Bade aus. Sie verkennt, daß die Frage nach Verantwortung und Schuld nicht nur eine moralische und politische Dimension hat, sondern sehr viel schlichter eine juristische und in diesem Zusammenhang darüber Auskunft gibt, wie Zahlungsverpflichtungen begründet werden und fortbestehen, wie sie eingeschränkt werden und fortfallen können. Es geht mit anderen Worten um einen Maßstab für Risikoverteilungen innerbalb von Vertragsbeziebungen. Daß dieser Maßstab nicht moralischen Kriterien folgt, zeigt sich im übrigen daran, daß häufig Zahlungspflichten in kommerziellen Beziehungen begründet werden oder wegfallen, ohne daß es auf Verschulden, Vorsatz, Fahrlässıgkeit oder böse Absicht ankäme. So läßt sich etwa ein Grundsatz aufzeichnen, nach dem Rechtssubjekten eine gewisse risikoreiche Tätigkeit gestattet wird, die zufälligen Folgen dieser Tätigkeit dann aber auch von diesen getragen werden müssen und nicht etwa von einem Dritten, der in die Risikosphäre gerät. 
Einem Beispiel für die Schuldnerbehandlung in den meisten Handelsrechten der industrialisierten Länder kommt für die Schulden-Debatte größere Bedeutung zu: Von der These ausgehend, daß unternehmerische Betätigung in der bürgerlichen Gesellschaft auch eine gesellschaftlich nützliche Angelegenheit ist, wird kommerziellen Schuldnern im Falle der Zahlungsunfähigkeit unter bestimmten Bedingungen die Möglichkeit eingeräumt, ein Zahlungsmoratorium oder gar einen teilweisen Schuldenerlaß von allen Gläubigern zu erlangen, um die bisherige Tärigkeit auf der Grundlage eines finanzierbaren Sanierungsplans fortzuführen und dies auch dann, wenn vor der Zahlungsunfähigkeit schweres Management-Verschulden vorlag.

Es ist also möglich, die Verantwortungsfrage von den moralischen Obertönen zu befreien und ihr gerade dadurch einen operationalen Sinn zu geben. Dieser Sinn bliebe auch dann bestehen, wenn die Idee von Schuldenerlaß und -moratorium sich weiter durchsetzen würde, weil aus ihnen Lehren für die Zukunft gezogen werden könnten und insbesondere die Länder nicht sich in der Stellung des Bittstellers und Abhängigen fühlen müssen, sondern der Erlaß Folge einer normalen juristischen Deduktion ist.

Als Beispiel für die hier vorgeschlagene Risikoverteilung zwischen Ländern der Dritten Welt und Consultants, Lieferanten, Finanzierungs- und Garantieinstitutionen möchte ich einen Fall anführen und ausführlich darstellen, der Anlaß zu einer Schiedsgerichtsentscheidung des ICSID (International Center for the Settlement of Investment Disputes: eine an die Weltbank angeschlossene Schiedsgerichtsstelle) gewesen ist. Im Jahre I971 schlug das multinationale Unternehmen K. der Regierung des Kamerun ein Projekt zum schlüsselfertigen Aufbau einer Kunstdüngerfabrik vor - auf der Grundlage einer von K. selbst ausgeführten Feasibility-Studie, die eine sehr hohe Rentabilität des Projektes auswies. K. hatte die Verpflichtung übernommen, die Anlagen zu liefern und die Finanzierung durchzuführen. Kamerun war verpflichtet, die Kredite zurückzuzahlen.

Die Fabrik erreichte niemals die break-even-Schwelle. Außerdem war das verwendete Material den klimatischen Bedingungen Kameruns nicht gewachsen. Dennoch wurde die Herstellung in der Endabnahme als ordnungsgemäß bestätigt. Die Fabrik hat in mehreren Anläufen nur eine geringe Kapazitätsauslastung erreicht und wurde nach einigen Jahren eingestellt, ohne daß die Prognosen der Feasibility-Studie jemals erreicht gewesen wären: eine neue Entwicklungs-Ruine war geboren. Die Verträge sahen freilich eine unbedingte Zahlungsverpflichtung vor, verstärkt durch die Ausstellung von Wechseln.

Die Schiedsrichter hatten in dieser Situation über die Zahlungsverpflichtung Kameruns zu entscheiden. Gemäß der Terminologie dieses Artikels: sie mußten zivilrechtliche Verantwortung zuschreiben und auf diese Weise das Risiko dafür zu verteilen, daß ein Projekt nicht in der geplanten Weise durchgeführt wird, daß es nicht rentabel ist, und daß es die zum Schuldendienst erforderlichen Fonds nicht aufbringen kann.

Die Schiedsrichter lehnten ausdrücklich ab, Aussagen über eventuelle Korruption oder betrügerische Absichten zu machen. Sie weigerten sich gleichfalls, »neue und besondere juristische Maßstäbe für schlüsselfertige Unternehmen anzuwenden, nur weil Projekte betroffen sind, welche die ökonomische und soziale Entwicklung eines Landes betreffen.$^{2}$ Sie gründeten im Gegenteil ihre Entscheidung auf allgemeine und traditionelle Prinzipien des Zivil- und Handelsrechts. In Anwendung

2 Dieses und die folgenden Zitate sind der Entschesdung vom 21 . Oktober 1983 entnommen und vom Verf. übersetzt; vgl. besonders auch Paulsson, Les Obligations des Partenaires dans un Accord de Développement Economıque: La Sentence Arbıtrale Cameroun c/ Klöckner, Revue de L'Arbitrage 1984, S. Ig ff. 
dieser Prinzipien gelangte die Mehrheit der Schiedsrichter zu der Überzeugung, daß Kamerun nicht verpflichtet sei, die Kredite zu honorieren. Was sind die Gründe für die Entscheidung? Die Schiedsrichter stellten fest, daß K. sich verpflichtet hatte, eine »betriebsbereite Fabrik «, nicht aber ein »Ensemble von Maschinen, Schläuchen und anderem Material « zu liefern. Der von K. tatsächlich gelieferte Komplex hätte niemals der Voraussage entsprechend gearbeitet. Die Endabnahme, welche die korrekte Ausführung bestätigt hatte, wurde zurückgewiesen, weil sie von einem Angestellten K's. durchgeführt worden war und „Gefälligkeitscharakter " aufwies. Der kamerunesische Beamte, der an der Abnahme teilgenommen und das Dokument mit unterzeichnet hatte, verfügte hingegen über keinerlei technische Kompetenz.

Der zentrale Grund jedoch für die Entscheidung der Schiedsrichter war, daß K. seinen Partner nicht mit der gebührenden Offenheit behandelt hatte: »Es handelte sich um ein gemeinsames Projekt, zu dessen Realisierung ein multinationales europäisches Unternehmen sich mit einem Entwicklungsland verbunden hatte. Die zu errichtende Fabrik war ein Beispiel moderner, importierter Technologie und Ingenieur-Wissenschaft. Kamerun verfügte über keinerlei Erfahrung im Bereich der Düngemittelproduktion. (...) Kamerun baute auf $\mathrm{K}$. bei der Lieferung alles für den Projekterfolg Norwendigen. (...) In einer derartigen Situation hat sich die Regierung K. ganz anvertraut. (...) Diese hat sich dem Partner gegenüber zwar nicht zu einer bedingungslosen Rentabilität verpflichtet, wohl aber zu einer weitgehenden Offenheit und Loyalität. (...) K. hat diese Verpflichtungen nicht erfüllt«. Die Nichterfüllung wurde darin gesehen, daß K. in der ersten Feasibility-Studie die Rentabilität der Fabrikation belegt hatte und dieses Ergebnis gegenüber dem Partner trotz einer bekannten negativen Marktentwicklung niemals in Zweifel zog: "Schon die erste Studie war vielleicht zu optimistisch und von dem Wunsch inspiriert, eine schlüsselfertige Fabrik zu liefern. Umso mehr widerspricht die Nicht-Mitteilung der Marktentwicklung dem Gebot der Offenheit zwischen Partnern. Dieses Fehlen von Zusammenarbeit war es, das der Regierung die Möglichkeit genommen hat, seine Meinung über die Realisierung des Projektes zu ändern.«

Das Schiedsurteil stellt fest, daß die Ausführung unvollständig und mangelhaft war. Im Einklang mit allgemeinen Prinzipien des Zivilrechts kommen die Schiedsrichter zu dem Ergebnis, daß die Teil-Nichterfüllung schwerwiegend genug war, um das synallagmatische Gleichgewicht vollständig zu stören und infolgedessen die Pflicht zur Gegenleistung aufzuheben. Die ausgestellten Wechsel, die im Prinzip einen eigenen Schuldgrund darsteilen, wurden in die Überlegung einbezogen: »Es ist offensichtlich, daß die beklagte Partei solche weitgehenden Verpflichtungen nur auf sich genommen hat, weil sie auf die Herstellung einer Fabrik vertraute, deren Produktion den berechtigten Hoffnungen entsprach, besonders auch eine Fabrik ohne schwere Korrosions-Schäden und andere schwere Konzeptions- und Konstruktionsfehler. Die Gleichheit zwischen den Parteien wäre eindeutig gestört, wenn $\mathrm{K}$. die Zahlung aus den Wechseln verlangen könnte. Diese Zahlung wäre eine offensichtliche Verletzung des vertraglichen Gleichgewichts. Die Verpflichtung der Republik Kamerun ist ohne Rechtsgrund, nachdem die versprochene Gegenleistung nicht erbracht worden ist. "

Welche Verallgemeinerungen lassen sich aus dieser Entscheidung ziehen? Zum einen sind zwei der großen Themen der Entwicklungsdebatte, nämlich der Technologie-Transfer und die Überschuldung materiell zusammengezogen worden. Der Fall kann als exemplarisch dafür gelten, in welcher Weise Feasibility-Studien in ihren Resultaten beeinflußt sind von Lieferanten-Interessen. Gleichzeitig belegt er in seinem Ablauf die Richtigkeit der soziologischen These, daß ein Unternehmen 
wesentlich nicht ein Ensemble von Maschinen und Anlagen ist, sondern ein Ensemble menschlicher Beziehungen, Kenntnisse, Marktbeziehungen und kultureller Eigenarten. Diese These wird im übrigen in der juristischen Doktrin geteilt, wenn es darum geht, den Mittelpunkt eines Unternehmens zu bestimmen. Sie trägt dies seı am Rande bemerkt - eine kritische Dimension in die Forderung nach Technologie-Transfer.

Darüberhinaus aber will ich eine entwicklungsspezifische Überlegung vorstellen, die ich gemeinsam mit G. Frankenberg entwickelt habe. ${ }^{3}$ Die Tatsache, daß die Schiedsrichter es ablehnten, in dem spezifischen Fall die besonderen Bedingungen der Nord-Süd-Beziehungen zu berücksichtigen, hindert ja nicht daran, diese Besonderheiten in allgemeine juristische Überlegungen und Konstruktionen einzubeziehen. Den Ausgangspunkt bildet die Tatsache, daß weder im Völkerrecht noch in nationalen Rechten jeder Vertrag unter allen Umständen erfüllt werden muß. Eine Reihe von Prinzipien engen die absolute Gültigkeit von Verträgen ein. Das gilt im Völkerrecht etwa für den Fall, daß tiefgreifende Änderungen in der faktischen Situation eintreten oder daß die Exekution des Vertrages einem der Partner unmöglich wird, wobei schon die Verletzung fundamentaler nationaler Interessen als Fall von Unmöglichkeit anerkannt wird. Beispielhaft läßt sich ein Zahlungsmoratorium anführen, das der jungen Bundesrepublik von den Siegermächten nach 1949 eingeräumt wurde, um den Prozeß der Staatskonsolidierung nicht zu gefährden. Eine andere Begrenzung, die wir in dem o. a. Essay präzisiert haben, bildet die Lehre von den unsittlichen Schulden, die typischerweise im Zusammenhang mit der Staatsnachfolge diskutiert worden ist. Ein Zitat des französischen Rechtsgeiehrten Sack, das für viele steht, soll den klassischen Zusammenhang dieser Doktrin beleuchten:

"Wenn ene despotısche Staatsgewalt enne Zahlungsschuld eingeht, die nicht für Zwecke und im Interesse des Staates liegt, sondern das despotische Regime stärken und die das Regime bekämpfende Opposttion niederhalten sollen; eıne solche Schuld ist sittenwidrig für die Bevölkerung des ganzen Staates. Diese Schuld bindet die Natıon nıcht; sie ist eıne Schuld des Regimes, enne persönliche Schuld der Staatsgewalt, von der ste enngegangen wurde, infolgedessen verfällt sie mıt dem Sturz des Regimes. Diese sittenwidrigen Schulden können nicht als verbındlich für das Staatsterritorıum angesehen werden, weil eine der Hauptbedingungen für die Gültigkett einer Staatsschuld fehlt, nämlich die, daß Staarskredire für die Bedürfnisse und im Interesse des Staates verwendet werden müssen. Die sittenwidrigen Schulden, von denen der Gläubiger weiß, daß sie entgegen den Interessen der Nation begründet und genutzt werden, binden diese nicht, falls sie sich von der Regierung befreien kann, oder doch nur in dem Maße, in dem sie einen materiellen Vorteil aus ihnen ziehen kann. Die Gläubiger haben gegenüber der Bevölkerung einen feindlichen Akt begangen: sie können nicht verlangen, daß eine Nation, die sich von einer despotischen Macht befre1t hat, die sirtenwidrigen Schulden bezahlt, die persönliche Schulden eben dieser Macht sind. In diese Kategorre fallen auch jene Zahlungsverbındlichkeıten, die eingegangen wurden im offensichtlichen persönlichen Interesse von Regierungsmitgliedern oder Gruppen, die der Regierung nahestehen und ohne Beziehung zu den Interessen des Staates sind ${ }^{4}{ }^{4}$

Wir haben diese Doktrin insoweit fortentwickelt, als der sittenwidrige Charakter sich nicht mehr auf die Person eines Herrschers bezieht, sondern die politischen und ökonomischen Fakten der Teilung der Welt in große ökonomisch ungleiche Blöcke in die Konstruktion einbezieht. Es wird bewußt auf ein Souveränitäts-Konzept verzichtet, das von der Völkerrechtsdoktrin des klassischen Imperialismus und seines Eurozentrismus entwickelt worden war, um die gleichwertige DominanzPolitik der europäischen Staaten zu sichern. Wir gehen also nicht von einer formalen Gleichheit aller Staaten aus, sondern nehmen politische und ökonomische Ungleich-

3 A. a. O. (FN. I).

4 Sack, Les effets des transformations des États sur leurs dettes publiques et autres obligations financières, 1927 (Übersetzung des Verf.). 
gewichte auf und halten es bei der Vertragsinterpretation entsprechend für jurtstzsch relevant, was faktisch ohnehin relevant ist, nämlich, daß ein Ungleichgewicht besteht zwischen einer Super- und/oder Mittelmacht einerseits und einem LLDC andererseits. Die Relevanz dieser Annahme folgt dann daraus, daß wir der in unzähligen Deklarationen einhellig wiederholten Auffassung aller Staaten folgen, der gemäß es heure eine Pflicht zur Entwicklung gibt, die sowohl die Staaten des Nordens als auch die Entwicklungsländer selbst bindet. Als prominente Fassung dieser Überzeugung sei Art. 55 der UN-Charta zitiert:

„Um jenen Zustand der Stabilität und Wohlfahrt herbeızuführen, der erforderlich ist, damit zwischen den Nationen friedliche und freundschaftliche, auf Achtung vor dem Grundsatz der Gleichberechtigung und Selbstbestimmung der Völker beruhende Beziehungen herrschen, fördern die Vereinten Nationen:

a) die Verbesserung des Lebensstandards, die Vollbeschäftigung und die Voraussetzung für wirtschaftlichen und sozialen Fortschritt und Aufstieg;

b) die Lösung internationaler Probleme wirtschaftlicher, sozialer, gesundheitlicher und verwandter Art, sowie die internationale Zusammenarbeit auf den Gebieten der Kultur und Erziehung;

c) die allgemene Achtung und Verwırklichung der Menschenrechte und Grundfreiheiten für alle ohne Unterschied der Rasse, des Geschlechts, der Sprache oder der Religion.»

Das Konzept einer formalen Souveränität wird auf diese Weise zumindest angereichert durch eines, das aktive Entwicklungspolitik als politische und rechtliche Verpflichtung vorschreibt und sich zum Ziel setzt, die gröbsten Ungleichgewichte zwischen den Staaten des Nordens und denen des Südens auszugleichen. Insoweit konkretisiert sich in der Tat ein Recht auf Entwrcklung, das allerdings manchen Regierungen (und dies auch Ländern der Dritten Welt) als lästige Pflicht erscheinen mag.

Diesen Aussagen wird entgegengehalten werden, daß die Diskussıon um »richtige" Entwicklung außerordentlich kontrovers ist, daß in konkreten Fällen Meinungsverschiedenheiten aus guten Gründen bestehen und daß es deshalb sehr schwierig ist, rechtliche Konsequenzen aus vagen und kontroversen Grundsätzen abzuleiten. Darauf läßt sich erwidern, daß Jurisprudenz seit jeher mit dem Dilemma fertig werden muß, allgemeine und vage Generalklauseln im Einzelfall zu konkretisieren, und daß bei allen Kontroversen im einzelnen doch eine gemeinsame Basis besteht, von der aus gewisse grobe Aussagen möglich sind. So mag mit Recht darüber gestritten werden, ob die schlüsselfertige Lieferung eines Zementwerkes in ein LLDC die Entwicklung des Landes vorantreibt; und es wäre falsch, die eine oder andere Entscheidung mit dem Geruch des Sittenwidrigen zu belegen. Nicht aber gestritten werden sollte auf der Basis der UN-Charta und anderer Grundüberzeugungen, daß der Aufbau eines Zementwerkes mit einer Kapazität von 200000 Tonnen in einem LLDC ohne Kalkvorkommen allzu einseitig den Lieferanten-Interessen des "Nordens" entgegenkommt und auch dann auf seinen sittenwidrigen Charakter zu untersuchen ist, wenn ein politischer Herrscher den Lieferanten einlädt, um einen technisch nicht überprüfbaren Traum von Zementautarkie zu erfüllen, der dann zerplatzt. Ebenfalls wird Einigkeit darüber bestehen, daß die Einfuhr von schweren Waffen in ein LLDC zur Niederhaltung einer inneren Opposition nicht einem Entwicklungsauftrag gerecht wird, oder daß die Einfuhr eines hochsensiblen, nur unter ganz bestimmten Umweltbedingungen, bei ständiger, hochqualifizierter Wartung betriebsbereiten Apparatur, so nützlich sie in Tokio sein mag, den Entwicklungsprozeß eines LLDCs eher hemmen als fördern wird.

Allgemeiner: Die Debatte um Entwicklung kennt wohl einen allgemeinen BasisKonsens, der genutzt werden kann, um über bestimmte Projekte ein Unwert-Urteil 
auf Entwicklungstauglichkeit zu fällen. Dieser Konsens ist für die Konkretisierung der akzeptierten Doktrin der sittenwidrigen Schulden fruchtbar zu machen.

Gemäß der von uns vorgeschlagenen Erweiterung der Doktrin auf die Erfordernisse einer konsensual-vernünftigen Entwicklung widerspräche es der Verpflichtung aller Staaten zu sozialer Gerechtigkeit und internationaler Solidarität, wenn Staaten für nachweislich unproduktive und entwicklungsfeindliche Investitionen und/oder Importe entweder Kredite in Anspruch nehmen oder öffentliche Kredite gewähren oder private Kredite mit öffentlichen Bürgschaften absichern. Private Gläubiger gehen ein hohes Risiko ein. Sie sind zwar nicht verpflichtet, zur Entwicklung des Landes beizutragen, müssen aber damit rechnen, $d a ß$ eine Regierung den Einwand erheben wird, eine bestimmte in der Vergangenheit eingegangene Zahlungsverpflichtung sei sittenwidrig und dementsprechend eine Zahlungsverpflichtung bestehe nicht. Eine derartige Umverteilung des Risikos durch Zuordnung einer juristischen Verantwortung, wie sie hier für zwei Bereiche vorgestellt worden ist, könnte zum einen einen Beitrag leisten zur Verringerung des Alt-Schulden-Bestandes. Wesentlicher erscheint jedoch der präventive Charakter einer solchen Doktrin: Wenn sie sich durchsetzt, steigt das Risiko für Lieferanten und Kreditgeber, und sie werden vielleicht mehr als bisher ihre technische Kompetenz einsetzen, um sich Gedanken zu machen über den Einfluß eines Projektes auf die Entwicklung der belieferten Länder.

\title{
In der NJW-Schriftenreihe
}

\author{
Hefi 41:
}

\section{Huber}

\section{Ausländer- und Asylrecht}

\section{Von Bertold Huber, Wiss. Angestellter an der Universität Frankfurt und Lebrbeauftragter an der Fachhochschule Frankfurt \\ 1983. LII, 256 Seiten. Kartoniert DM 39,- ISBN 3-406-09842-8}

Die rechtliche Stellung der in der Bundesrepublix lebenden Auslānder bildet seıt Jahren eınen der Schwerpunkte der täglichen Behörden-, Genchtsund Anwaitspraxis und hat zu einem enormen Anstieg einschlāgiger Rechtsprechung und Literatur geführt. Infolgedessen fällt es immer schwerer, den Überblick über Stand und Entwnckiung des Auslānderrechts zu erhalten und zu bewahren. Das vorliegende Werk bietet eıne systematische Gesamtdarstellung des geltenden Ausländer- und Asylrechts.

\author{
Ausfïhrlich and umfassend werden u.a. eriäutert \\ - Ehegatten- und Familiennachrug \\ - «scheineben \\ - Verbot der Arbertsaufnahme durch die Auslãn- \\ derbebörde \\ - Verfestigung des aufenthaltsrechtlichen Status \\ - Ausweısung wegen Straffālligkeit \\ - Freızügigkett für Angehönge der EG-Staaten \\ - Asylgründe \\ - Zugang zum deutschen Arbettsmarkt \\ - Einstweiliger Rechtsschutz \\ Das Buch wendet sich in erster Linie an Richter, \\ Rechtsanwälte, Ausländerbehörden und Arbeits- \\ ämter, austāndische Botschaften und Konsulate, \\ Sozialarbeiter und Sozialberater für ausländische \\ Arbeitnehmer, Woblfahrtsverbände und Initiativ- \\ gruppen.
}

\section{Verlag C.H.Beck München}

